\title{
¿Quién tiene Derecho a la Ciudad? Jóvenes Lesbianas en Brasil y Cataluña desde las Geografías Emocionales e Interseccionales
}

\author{
Who has the Right to the City? Young Lesbians in Brazil and Catalonia \\ from Emotional and Intersectional Geographies
}

\section{Quem tem Direito à Cidade? Jóvens Lésbicas no Brasil e na Catalúnia a partir das Geografias Emocionais e Interseccionais}

\author{
Maria Rodó-de-Zárate \\ Universidade Estadual de Ponta Grossa, Brasil \\ maria.rodo.zarate@gmail.com
}

\section{Resumen}

El Derecho a la Ciudad se ha concebido como un derecho humano fundamental de aquellas personas que habitan las ciudades. Pero ¿quién tiene este derecho? Con la investigación desarrollada en Manresa (Cataluña) y en Ponta Grossa (Brasil), dos ciudades medianas no metropolitanas, analizo las negociaciones espaciales de jóvenes lesbianas en su vida cotidiana. Desde una perspectiva interseccional y emocional examino cómo sus diferentes posiciones condicionan y limitan su acceso a la ciudad. Con este estudio pretendo mostrar cómo el Derecho a la Ciudad debe conceptualizarse teniendo en cuenta las discriminaciones por razón de orientación sexual, la dimensión afectiva de la desigualdad, las múltiples opresiones que interseccionan y la (no)separación entre espacios públicos y privados.

Palabras-Chave: Sexualidades; Feminismo; Juventud; Interseccionalidad; Espacio PúblicoPrivado.

\begin{abstract}
The Right to the City has been conceived as a fundamental right of the city's inhabitants. But, who has this right? From a research conducted in Manresa (Catalonia) and Ponta Grossa (Brasil), two medium and non-metropolitan cities, I analyze the spatial negotiations of young lesbians in their everyday life. From an intersectional and emotional perspective I examine how their different positions condition and limit their access to the city. With this study I aim to show how the Right to the City must be conceptualized, taking into account discriminations due to sexual orientation, the affective dimension of inequality, the multiple oppressions that intersect and the (non)separation of public and private spaces.
\end{abstract}

Keywords: Sexualities; Feminism: Youth; Intersectionality; Public-Private Space. 


\section{Resumo}

O direito à cidade foi concebido como um direito humano fundamental para as pessoas que a habitam. Mas, quem tem este direito? Com a pesquisa realizada em Manresa (Catalúnia) e Ponta Grossa (Brasil), duas cidade médias não metropolitanas, analiso as negociações espaciais de jovens lésbicas em sua vida cotidiana. A partir de uma perspectiva interseccional e emocional, examino como suas diferentes posições condicionam e limitam seu acesso à cidade. Com este estudo pretendo mostrar como o direito à cidade deve ser conceitualizado, tendo em consideração as discriminações por orientação sexual, a dimensão afetiva da desigualdade, as múltiplas opressões que intersccionam e a não separação entre os espaços públicos e privados.

Palavras-Clave: Sexualidade; Feminismo; Juventude; Interseccionalidade; Espaço Público-Privado.

\section{Introducción}

El derecho a la ciudad es más que la libertad individual de acceder a los recursos urbanos: es el derecho a cambiarnos a través de cambiar la ciudad. Es, además, un derecho común más que individual, ya que su transformación depende inevitablemente del ejercicio de un poder colectivo para remodelar los procesos de urbanización. Quiero defender que la libertad de hacer y rehacer nuestras ciudades y a nosotrxs mismxs es uno de los derechos humanos más preciados y sin embargo es también uno de los más descuidados (HARVEY, 2008, p. 23). ${ }^{1}$

David Harvey en esta cita muestra la relevancia del derecho a la ciudad como un derecho común, 'el más preciado' pero que pocas veces se toma en cuenta. Lefebvre (1968) basaba el derecho a la ciudad en el derecho a la apropiación y a la participación (PURCELL, 2002). Aquí analizaré hasta qué punto esta conceptualización permite dar cuenta de determinadas discriminaciones y exclusiones en el espacio público.

Centrándome en la investigación empírica realizada con lesbianas jóvenes de dos ciudades medianas no metropolitanas de Brasil (Ponta Grossa - Paraná) y Cataluña (Manresa - Barcelona), pretendo mostrar las limitaciones que sufren en su acceso al espacio público. A pesar de que hoy en día son numerosos los estudios sobre gays y lesbianas en espacios urbanos en el ámbito internacional, la relación entre mujeres jóvenes y su sexualidad es un tema prácticamente ausente en geografía. El hecho de tomar un grupo poco estudiado $y$ atravesado por diversas opresiones permite examinar el Derecho a la Ciudad desde otra perspectiva, que en este caso es interseccional (por sus diversas posiciones en estructuras de poder como el género, la edad o la clase social) y emocional (focalizando en los sentimientos que tienen en determinados lugares). A través de los Relief Maps o Mapas de Relieves de la Experiencia, una metodología empírica y analítica que he desarrollado para estudiar la interseccionalidad de una forma visual y espacial, pretendo mostrar cómo la sexualidad, siempre en relación con otras identidades, es para estas jóvenes lesbianas un factor fundamental que limita su acceso a la ciudad.

En el siguiente apartado situaré este estudio en relación a la literatura existente 
para posteriormente detallar la metodología usada. A continuación analizaré las limitaciones del Derecho a la Ciudad en función a cuatro cuestiones: la violencia heterosexista directa, la dimensión afectiva de la discriminación, el espacio privado como fuente de discriminaciones y las múltiples identidades como límites de acceso. Finalizaré con las conclusiones.

\section{Geografías en la Encrucijada}

Esta investigación se sitúa en medio de las geografías feministas, de las sexualidades y de la juventud, dialogando con las geografías urbanas y del espacio público. Este lugar en la encrucijada de diferentes ámbitos de estudio permite abordar cuestiones fundamentales en la investigación geográfica, como el derecho al espacio o la justicia espacial, desde perspectivas que a menudo permanecen invisibilizadas.

Estudiar las experiencias del espacio urbano de mujeres jóvenes lesbianas de dos ciudades no meteropolitanas implica focalizar el estudio desde una perspectiva interseccional. Si bien las lesbianas son un grupo bastante estudiado en las geografías urbanas, a menudo son solo las blancas, adultas y de ciudades metropolitanas las que se incluyen en tales investigaciones. En las geografías feministas, se tiende a tener una perspectiva heteronormativa, $y$ en las geografías de las sexualidades predominan los estudios sobre hombres gays (BROWNE y FERREIRA, 2015). Lo mismo sucede con el estudio de la juventud. Es difícil encontrar trabajos que se centren en jóvenes con sexualidades disidentes (SCHROEDER, 2012), y menos aún si son mujeres. Existen estudios que examinan las relaciones entre sexualidad y género (PEAKE, 1993; SILVA, 2009; BROWNE, 2007; PODMORE, 2001, 2006; SANTOS SOLLA, 2002; TAYLOR, 2010; PRITCHARD et altri, 2002;
ROTHENBERG, 1995; VALENTINE, 1993) y hay algunos sobre jóvenes gays y lesbianas en espacios públicos (FERNANDES CARVALHES et altri, 2011; BARCELOS SOLIVA, 2011), en relación con el rol de los espacios de 'ambiente' en su proceso de 'salida del armario' (VALENTINE y SKELTON, 2003) y sus negociaciones identitarias en internet (DOWNING, 2013). A pesar de esto, faltan estudios que analicen las experiencias de jóvenes lesbianas teniendo en cuenta sus múltiples opresiones y negociaciones. Como muestra Harding (2004, p. 7-8), "la desventaja política se puede convertir en una ventaja epistémica, científica y política". Tomar este grupo en los márgenes para el estudio de un tema central como el Derecho la ciudad puede pues aportar nuevas visiones y perspectivas que son invisibles desde el centro.

Así, la interseccionalidad, como concepto que muestra la necesidad de tener en cuenta las múltiples interconexiones entre diferentes estructuras de poder (COLLINS, 1998; DAVIS, 2009; MCCALL, 2005) aparece como una perspectiva necesaria para analizar la experiencia vivida, que pretende además evitar la exclusión de los grupos que acostumbran a quedar en los márgenes. Taylor (2010) muestra como la interseccionalidad aparece como un paradigma complicado pero útil para los estudios de sexualidades, ya que permite tener en cuenta la sexualidad en el estudio de otras dimensiones de poder y tener a estas en cuenta en los estudios de sexualidad. La necesidad de abordar las cuestiones de sexualidad desde una perspectiva interseccional también ha sido puesta de manifiesto en investigaciones en Brasil y en el estado español (ver SILVA y ORNAT, 2014; PLATERO, 2012).

En relación a las sexualidades disidentes y el espacio público, se ha mostrado como estos espacios se construyen como 
heteronormativos a través de la repetición de determinadas prácticas (BELL y VALENTINE, 1995; Bell et altri, 1994; BINNIE, 1997, VALENTINE, 1993) a la vez que actúan también como espacios de liberación sexual cuando ofrecen anonimato. Concretamente, las investigaciones sobre las experiencias de lesbianas en espacios urbanos se centran en sus prácticas en espacios comerciales, políticos, residenciales o de ocio (ROTHENBERG 1995; VALENTINE 199; PODMORE, 2006; CATTAN y CLERVAL, 2011). Una de las cuestiones que ha suscitado más interés ha sido la invisibilidad de las lesbianas en el espacio público, como también el desarrollo de los barrios gays y espacios comerciales. Uno de los motivos por los que estos temas se han centrado en las investigaciones, puede ser por el hecho de que los estudios sobre lesbianas se han dado en ciudades grandes. Como afirma Muller Myrdahl (2013), las lentes con las que normalmente se analizan las vidas 'queer' tienden a crear jerarquías entre ciudades grandes y ciudades pequeñas, reforzando el imaginario popular de que las pequeñas son más conservadoras y deben compararse con las metropolitanas. En esta investigación pretendo evitar tanto la comparación con jóvenes gays como con ciudades mayores. Considero las prácticas y negociaciones de las chicas lesbianas en relación con el entorno heterosexista y adultista en el que viven, y no en relación con la medida masculina de la visibilidad, y comparo dos ciudades medianas para ver qué semejanzas y diferencias se encuentran. Así, mi aproximación pretende analizar las experiencias de jóvenes lesbianas en el espacio urbano para ver en qué medida tienen limitado su acceso al espacio público, condición básica para el acceso a la ciudad.

\section{Metodología}

El trabajo empírico se ha realizado en
Manresa (Cataluña) y Ponta Grossa (Brasil). Manresa es una ciudad situada al noreste del Estado español, a unos 50 kilómetros de Barcelona, la capital de Cataluña. Es una ciudad mediana de más de 70.000 habitantes. Es la capital de la comarca del Bages (una división administrativa) $\mathrm{y}$, a pesar de que fue una importante ciudad industrial, hoy en día su economía se focaliza en el sector de servicios. Durante los años 1950 y 1960, la ciudad recibió una gran cantidad de inmigración proveniente del sur del Estado. Sin embargo, desde los años 1990 la mayoría de inmigrantes provienen del norte de África y de América Latina.

Ponta Grossa es una ciudad brasilera que se encuentra a 103 kilómetros de Curitiba, la capital del estado de Paraná. Tiene una población de más de 300.000 habitantes $\mathrm{y}$, junto con Curitiba, es un polo industrial del estado de Paraná. Su composición étnica es diversa, pero mayoritariamente está compuesta por descendientes de inmigrantes europeos. Inicialmente por portugueses, y desde principios del siglo XX por alemanes y eslavos.

La comparación entre estas dos ciudades medianas, con roles parecidos en sus respectivos contextos, permite un análisis que se aleja de las comunes comparaciones entre ciudades pequeñas y medianas con grandes metrópolis. Siguiendo las investigaciones de Muller Myrdal (2013), parto de la base que no es la medida de la ciudad lo que hace más o menos fácil la expresión de las sexualidades disidentes sino una variedad de contingencias geográficas que producen determinadas normas sociales.

Centraré mi análisis en las 16 entrevistas llevadas a cabo con jóvenes lesbianas, de 16 a 25 años, siete en Manresa y nueve en Ponta Grossa. A pesar de que la muestra es reducida, no pretende ser generalizable sino que el objetivo es entender de qué forma su acceso a la ciudad está condicionado por sus 
diferentes posiciones. Para el análisis interseccional he considerado sus posiciones de género, sexualidad, edad, clase social y etnicidad. Cuestiones como el estatus familiar, el barrio donde viven, su religión o las actividades formativas y laborales también se han tenido en cuenta.

Las entrevistas semi-estructuradas consistieron en una primera parte donde las participantes podían mostrar sus experiencias cotidianas en el espacio público, como los lugares que frecuentan, sus itinerarios, sus lugares favoritos y los motivos por los que sienten determinadas experiencias en ellos. Después de la entrevista, se les pidió que realizaran su propio Relief Map. Estos son una herramienta desarrollada durante mi investigación doctoral sobre las Geografías de la Interseccionalidad con la que se pretende facilitar el estudio geográfico sobre las dinámicas interseccionales de opresión y privilegio (RODÓ-DE-ZÁRATE, 2014). Su elaboración implica el desarrollo de diversos pasos (ver RODÓ-DE-ZÁRATE, 2014b) que ayudan a las participantes a reflexionar sobre los lugares que utilizan y las emociones que les generan, siempre procurando que tanto la forma como sus diferentes posiciones de género, sexualidad, edad, clase y etnicidad que condicionan su experiencia, sean visibles. El último paso, la elaboración por parte de las participantes de su propio Relief

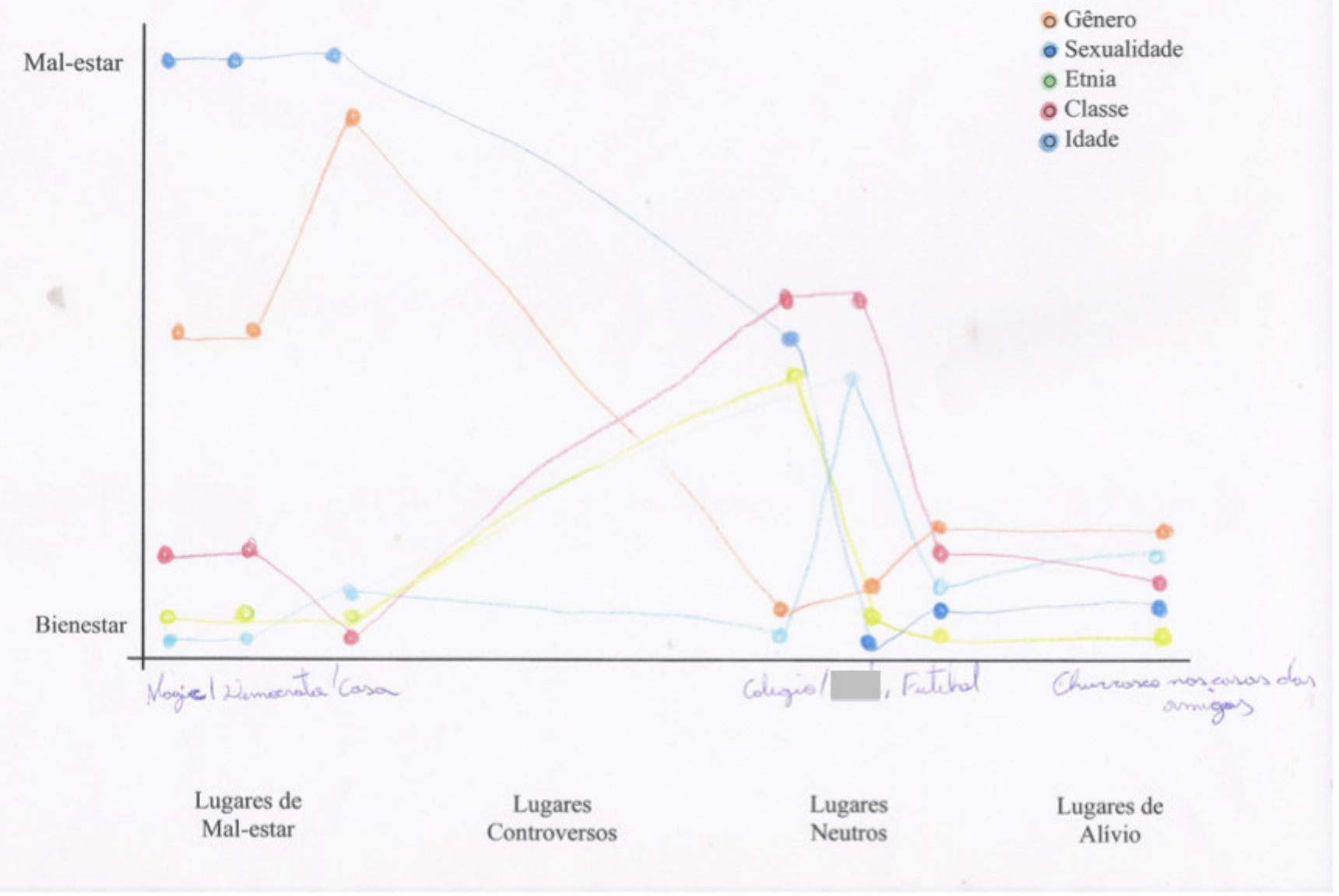

Figura 1 - Relief Map de Denise ${ }^{2}, 18$ años. Ponta Grossa. 
Map conlleva el dibujo que una representación visual de su experiencia en los espacios (Figura 1).

Los puntos situados en la parte superior muestran un malestar agudo en relación con una identidad concreta $\mathrm{y}$ en un lugar determinado. En cambio, los punto situados en la parte inferior muestran el bienestar. Los lugares también se distribuyen de izquierda a derecha, según sean de mayor malestar o bienestar, hecho que permite llamar a estas figuras 'mapas', ya que sus experiencias interseccionales se representan de forma visual sobre una distribución espacial. ${ }^{3}$ Así, el material empírico usado, son las narrativas y la información recogida a través de los Relief Maps.

\section{¿Quien tiene derecho a la ciudad?}

Henri Lefebvre (1968) entendía en derecho a la ciudad como el derecho de aquellos que la habitan (inhabit), hecho que tiene muchas implicaciones para la misma concepción de la ciudadanía, conllevando una reconceptualización de ésta. Este derecho se basa, por un lado, en apropiarse del espacio urbano en el sentido del derecho a usarlo de forma plena y completa y, por el otro, en el derecho a la participación, a tomar un rol central en el proceso de toma de decisiones en relación a la producción del espacio urbano (PURCELL, 2002). Esta concepción ha sido criticada por geógrafas feministas porque no tiene suficientemente en cuenta las diferencias individuales y colectivas y porque no incluye en su análisis las relaciones patriarcales de poder (ver FENSTER, 2005).

En esta sección analizaré las limitaciones en el derecho a la ciudad de jóvenes lesbianas en Manresa y Ponta Grossa en base a cuatro elementos que se detallan a continuación. Básicamente hablaré de acceso al espacio relacionándolo con el derecho a la apropiación, al uso. En relación al derecho a la participación, no entraré a analizar detalladamente los procesos de participación formales ya que hasta los más básicos, como el derecho a voto, están prohibidos para muchas de las mujeres entrevistadas, ya que no alcanzan la mayoría de edad.

\section{Violencias heterosexistas como límite de acceso}

Una de las cuestiones que aparece como limitación más directa y visible en las narrativas de las entrevistadas es la negación directa a usar un lugar:

Un día estábamos desayunando en un bar y ni la besé ni nada. Sólo nos dábamos la mano y hablábamos. iY el tío del bar nos invitó a salir!! Nos fuimos sin pagar. (Elisa, 23 años, Manresa)

Essa é uma casa noturna também só que é muito ruim. Lá sim se você é um gay os próprio segurança já barram (Larissa, 18, Ponta Grossa).

En estas citas se aprecia como por el hecho de ser lesbianas se limita su acceso a determinados lugares. En el caso de Elisa, ella y su pareja son echadas de un bar por estar juntas $\mathrm{y}$, como Larissa cuenta, en determinados lugares de ocio ni siquiera las dejan entrar si se percibe que son lesbianas. En estos casos el límite de acceso es directo y constituye una clara discriminación por razón de orientación sexual. En otros casos, como el de Sílvia, son los comentarios y la violencia homofóbica los que indican la limitación.

Son los típicos comentarios de 
'¡lesbiana de mierda!' Y no sé qué más. Hasta tuve una pelea. [...] Llamaron 'puta tortillera' a mi amiga y le di un puñetazo (Sílvia, 17, Manresa)

En estos casos, a pesar de la preocupación que suponen este tipo de situaciones, ellas, de una forma $\mathrm{u}$ otra, responden a las agresiones, ya sea no pagando al salir del bar como acto de protesta (Elisa), o responder con contundencia a comentarios insultantes (Sílvia). Si bien estas respuestas muestran su rechazo a estos comportamientos, el recuerdo de estas situaciones y de otras presenciadas funciona como un recordatorio sobre los límites de lo que pueden hacer o lo que no.

Tem aquele lugar assim que, fica meio assim de faze porque as pessoas começam a olhar de uma forma diferente. A gente tem medo né por ser repreendida por tá ali cá pessoa que a gente gosta. Uma pessoa chega e xinga a gente ou mesmo quere bate na gente assim. (Larissa, 18, Ponta Grossa).

Ah eu já sofri contei um caso pra ela que tava no ônibus vindo embora, tinha duas amigas nossa. Elas tavam no banco da frente eu tava atrás e um homem sentado né e elas se abraçando, se beijando dentro do onibus e dai tinha uma mulher e a mulher falo "é coisa mais ridícula se fosse filha minha eu dava na cara, matava" bem assim, dai o rapazinho do lado "é mas se a gente faze isso a gente é homofóbico, preconceito (Carolina, 18, Ponta Grossa).

Ya sea porque, como en el caso de Carolina, se presenció una actitud homofóbica contra otras personas o contra ellas mismas, el miedo del que habla Larissa se encuentra presente en todas sus narrativas. Buscan los límites y analizan cada situación, para saber hasta qué punto puede ser peligroso o no mostrarse afecto. La homofobia en el espacio público por parte de personas anónimas, deviene uno de los límites de acceso al espacio público para ellas. Su estrategia más común es la de gestionar su identidad sexual a través del 'passing', es decir pasar como heterosexuales escondiendo su sexualidad y evitar así agresiones ${ }^{4}$. Las miradas reprobatorias y el miedo a posibles agresiones, son parte también de este espacio público y, por tanto, de sus vidas.

Con estos ejemplos, se muestra como el heterosexismo puede ser una fuente directa que impide el acceso a ciertos lugares. $\mathrm{Y}$ no es solo la negativa directa de estar en un lugar, sino también la forma como estas acciones condicionan el uso de los espacios por miedo a que puedan volver a repetirse. A pesar de estas restricciones directas, por contra, en sus narrativas también aparecen lugares creados como alternativa a la falta de espacios donde no sentirse fuera de lugar:

No shopping tem muito, por causa de ser lugar mais familiar né shopping é mais complicadinho. Ah mas tem banheiro lá. Se acontece, se a gente quando deseja fica com menina fica no banhero. Banheiro ou no cinema as vezes (Tatiana, 24, Ponta Grossa)

Um lugar que é conhecido é a estação, nossa lá é o principal, parada das lésbica ali. Ninguém fala nada porque ali é nosso digamos assim, ali a gente... nóis já dominamo aquela área. Agora é toda sexta-feira, final de semana é o 
¿Quién tiene Derecho a la Ciudad? Jóvenes

lesbianas en Brasil y Cataluña desde las

geografías emocionales e interseccionales

encontro do pessoa (Larissa, 18, Ponta Grossa).

Como muestran estas citas, contra las limitaciones de acceso a determinados lugares, ellas misma crean sus propios espacios de bienestar en el espacio público. La 'estación' (en referencia al edificio y la plaza donde antes estaba la antigua estación de trenes) es para Larissa un lugar 'dominado' por lesbianas. Ellas lo frecuentan, lo usan y con su presencia y sus acciones lo impregnan de una identidad propia. Como afirma Lane (2015) el hecho de compartir posiciones de género, sexualidad y edad con otras lesbianas contribuye a generar bienestar. En el caso de los baños del centro comercial, como comenta Tatiana, las restricciones que encuentran en los espacios comerciales son transgredidas usando un espacio concreto, el baño, como lugar donde estar en pareja, sin miedo a posibles represalias ni miradas reprobatorias. En este sentido, a pesar de las limitaciones de acceso que sufren, se visibiliza su capacidad para crear nuevos lugares que cuestionan la norma heterosexual. A diferencia de estudios que muestran la capacidad de lesbianas de intervenir en el espacio público a través de manifestaciones organizadas que demandan el Derecho a la Ciudad, este tipo de ocupaciones del espacio público son individuales y cotidianas, pero también contribuyen a la producción del espacio urbano. Así, su participación en esta producción, si bien no es ni formal, no está reconocida y se desarrolla desde los márgenes y en cotidianidad, forma parte también de la construcción del espacio urbano y podría entenderse como una forma de demanda de Derecho a la Ciudad.

Geografías Emocionales como

Indicadores de Desigualdad
No vas al barrio antiguo de Manresa y te besas o te das la mano porque claro, no sabes lo que puede pasar. Es solo... Metamorfosearte con el entorno. (Neus, 23 años, Manresa).

No shopping as pessoas olham bastante ficam mesmo sem não vou pegar na mão não vou abraçar não vou beijar, as pessoas olham bastante, parecem que notam, bastante... só conversando pela proximidade as pessoas acabam olhando (Natalia, 24, Ponta Grossa)

Eu me sentia normal por que a gente não demonstrava assim que era um casal, mais sempre...eu me sentia normal mais preocupada é me policiando pra não demonstra pros outros não ficarem sei lá olhando ou comentando, sempre a gente tomava esse cuidado assim. (Andreia, 26, Ponta Grossa)

A moça que eu namorei ela queria sair, ir no shopping compra assim, fazer um lanche, e ela queria mostra assim tipo abraçar sabe, anda de mãos dadas e eu nunca aceitei assim por que como eu falei sempre tem um conhecido (Luciana, 27, Ponta Grossa)

Más allá de las negativas directas, como muestran estas citas, estas chicas usan diferentes espacios de la ciudad, tanto en Manresa como en Ponta Grossa. Van de compras, pasean por la calle, van a un bar. Usan el espacio público al menos en un sentido estricto, en el sentido de que están en él y realizan algún tipo de actividad. Pero a la luz de estas citas, ¿es suficiente analizar su acceso a través de un enfoque reducido del 
'uso'? Sin entrar a analizar concretamente cada cita, se aprecia claramente que la forma en cómo están en estos espacios es fundamental para su experiencia: viven los espacios con temor, incomodidad y limitaciones. Esta perspectiva emocional, permite una aproximación al acceso y, por tanto, al derecho a la ciudad, que supera análisis cuantitativos sobre quién usa qué espacios y incide en la complejidad del acceso a través de la experiencia vivida de los espacios. Como argumentan Bondi y otros (2007) sobre las geografías de las emociones, a pesar de que se remarca el aspecto "emocional de las alarmantes adversidades e injusticias causadas por desigualdades y opresiones, generalmente no se investiga la forma como las emociones también contribuyen a su sostenimiento".

Desde esta perspectiva emocional, el análisis de sus narrativas muestra claramente los malestares que sufren en el espacio público, básicamente por su sexualidad. Como se ha podido ver en las citas anteriores, existen malestares en mucho de los lugares que usan en su vida cotidiana y hay algo en común que todas temen: las miradas reprobatorias, discriminaciones y agresiones. $\mathrm{Y}$ en todas ellas coincide que el tipo de acción penalizada, es la misma: besos, abrazos y muestras de cariño. Como he mostrado en trabajos anteriores (RODÓDE-ZÁRATE, en prensa), el hecho de no poder tener relaciones amorosas en determinados espacios constituye un tipo de desigualdad, la afectiva, que está fuertemente condicionada en este caso por el heterosexismo. Más que la sola presencia de sus cuerpos, es el hecho de tener un determinado tipo de actitud, cariñosa, la que parece que puede desatar la reacción homofóbica. Así, son este tipo de actitudes las que están fuertemente sancionadas y con las que estas mujeres conviven constantemente. Como comenta Neus, hay barrios concretos en su ciudad, donde sabe que no podrá ir tomada de la mano con su pareja. O Luciana, que negocia este tipo de acciones con su pareja.

Estos malestares que sufren en diferentes lugares se pueden ver también claramente en los Relief Maps. En los dos que se muestran a continuación se aprecia claramente cómo la línea azul claro (la que muestra las experiencias de bienestar o malestar en relación a su sexualidad) está acentuada en la mayoría de lugares que usan en su vida cotidiana (Figuras 2 y 3). En muchos de ellos no es solo la sexualidad lo que causa malestar, sino que en muchos de los lugares el género o la edad también están acentuados. Estos casos muestran cómo las emociones dan forma a los lugares (DAVIDSON y otros, 2007) y cómo estas emociones nos indican formas concretas de discriminación que a menudo quedan invisibilizadas.

A pesar de que en los discursos homofóbicos acostumbra a estar presente la idea de que la homosexualidad se acepta mientras no se muestre en espacios públicos, las imágenes de la heterosexualidad son omnipresentes en los espacios públicos de tal forma que se convierten en 'invisibles' (KUHAR, 2011). Así, y como argumenta Valentine (1993), la heterosexualidad no solo está definida por actos sexuales privados sino que se expresa en una gran variedad de formas, está institucionalizadas y se celebra en los espacios públicos. Mientras las personas heterosexuales toman la calle como si fuera un espacio heterosexual, "las lesbianas ejercen una auto vigilancia constante, controlando cómo van vestidas, su comportamiento y deseos para evitar la confrontación" (VALENTINE, 1996, p. 148). El hecho de que este tipo de acciones, como besarse o darse la mano, esté 'prohibida' muestra una importante desigualdad en la forma como se vive la ciudad. Este espacio público construido como heteronormativo y 
¿Quién tiene Derecho a la Ciudad? Jóvenes

lesbianas en Brasil y Cataluña desde las

geografías emocionales e interseccionales

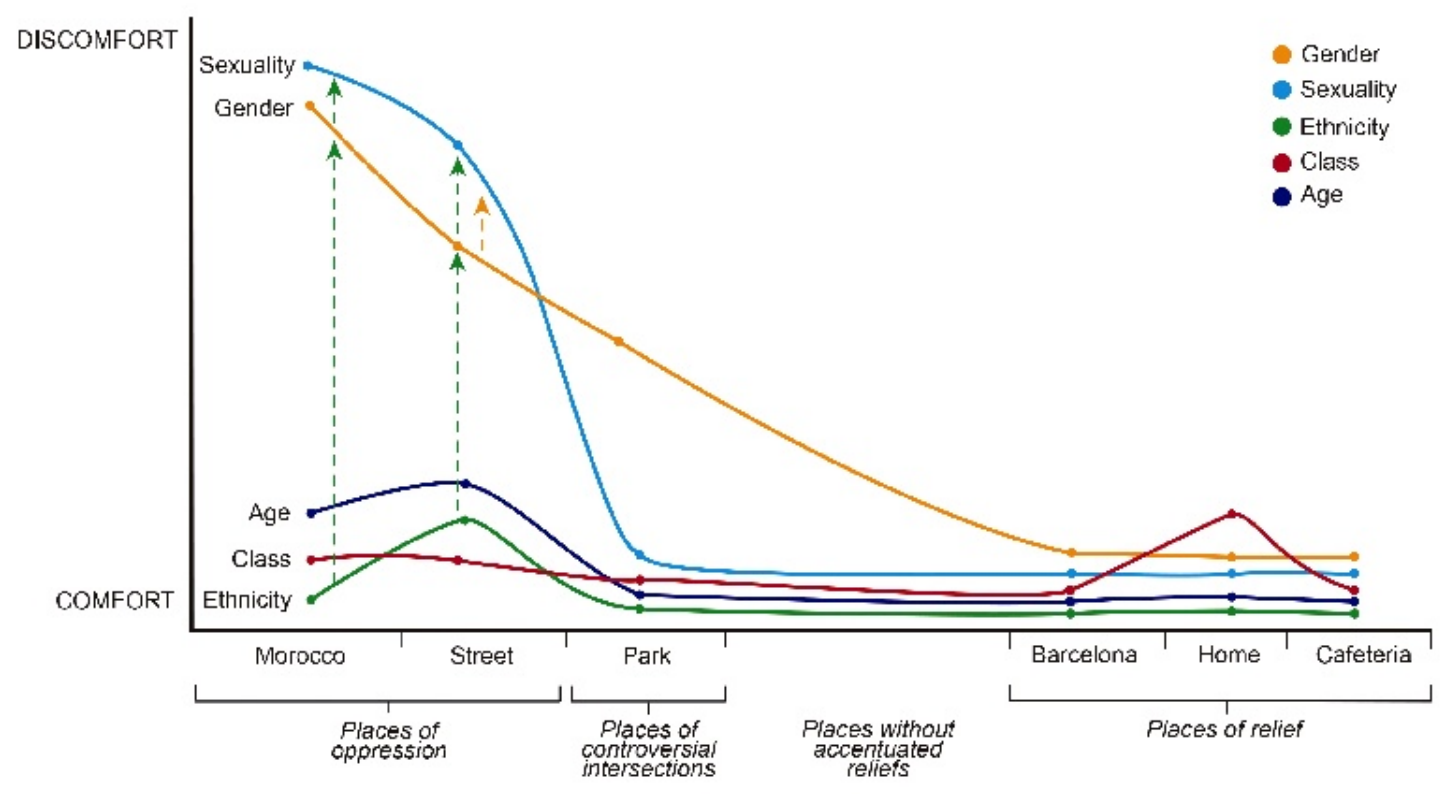

Figura 2: Relief Map Aya (lesbiana de origen Marroquí de 24 años que vive con su pareja. Manresa)

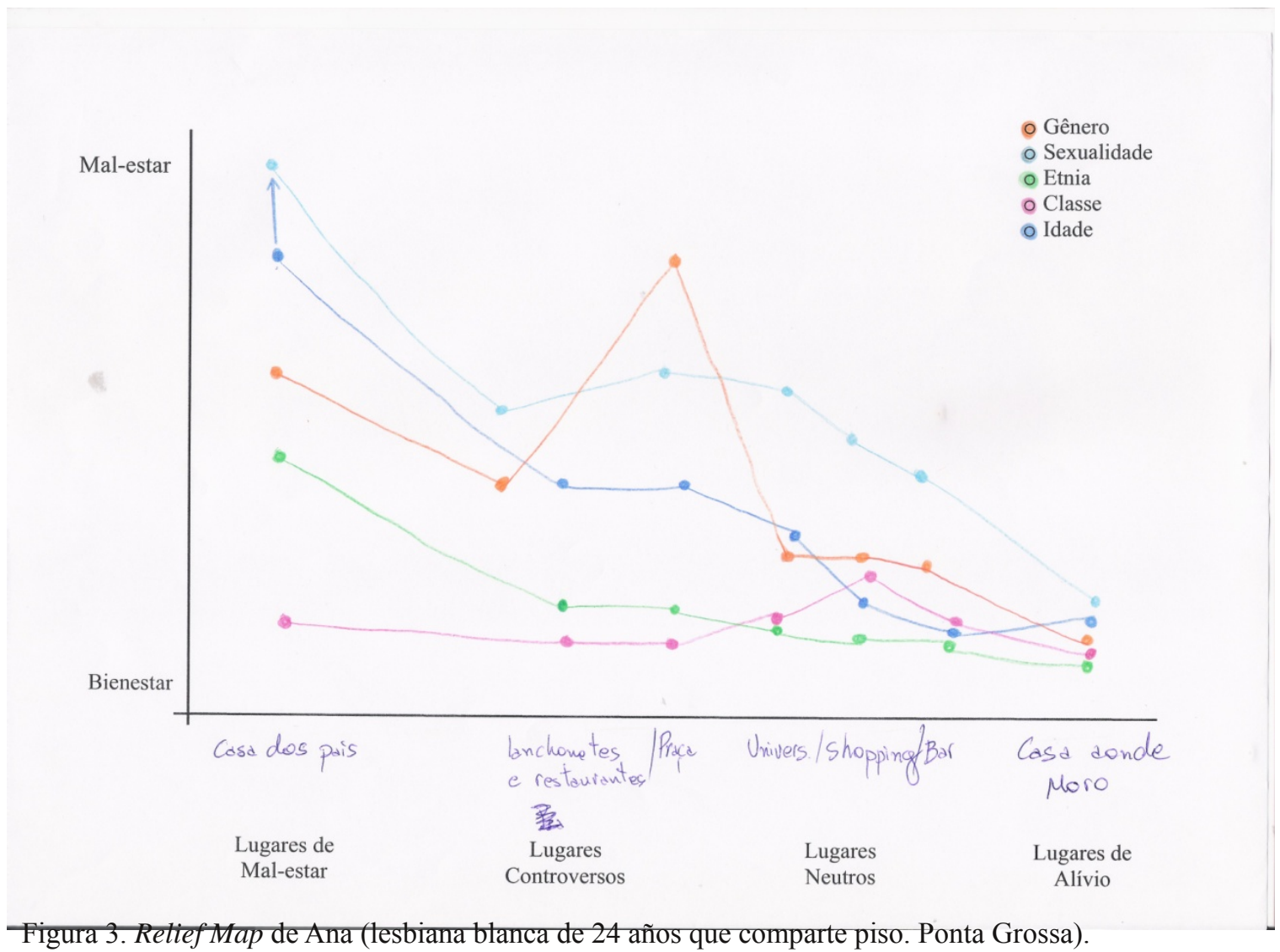

Maria Rodó-de-Zárate

Revista Latino-americana de Geografia e Gênero, Ponta Grossa, v. 7, n. 1, p. 3 - 20, jan. / jul. 2016. 
¿Quién tiene Derecho a la Ciudad? Jóvenes

lesbianas en Brasil y Cataluña desde las

geografías emocionales e interseccionales

(re)producido como si preexistiera lo heterosexual (BELL y VALENTINE, 1995; BELL et altri, 1994; BINNIE, 1997, VALENTINE, 1993) se sostiene a través de cuestionamientos que "provocan malestar y hacen que las lesbianas se sientan 'fuera de lugar' en los espacios cotidianos" (VALENTINE, 1996, p. 148).

Así, se muestra que no es suficiente analizar el uso como parte esencial del Derecho a la Ciudad, si no se examina desde una perspectiva emocional. Usar o no usar un espacio no da suficiente información sobre las limitaciones, ya que la forma cómo se está en estos espacios, las emociones y sentimientos que provocan, son también fundamentales para apreciar las posibilidades de acceso. Tener miedo mientras se está en un lugar, no poder abrazar a tu pareja o estar en constante alerta, constituye un límite al derecho a la ciudad que en este caso está fuertemente condicionado por la heteronormatividad en los espacios públicos.

\section{La Casa como Parte de la Ciudad}

Eu sou dependente deles por isso que eu não me assumo assim porque eu tenho muito medo deles cortarem (Larissa, 18, Ponta Grossa).

No puedo [mostrar mi sexualidad] en el espacio público. Mis padres no lo saben y me tienen muy reprimida. Se enteraron hace un año pero les dije que no y ahora no lo saben. Si se enteraran me moriría. Me reprimieron mucho. No me dejaban salir de casa. Me quedé en casa y en una semana perdi tres kilos. (Adriana, 17, Manresa).

Agora a relação tá, tá evoluindo, tá indo mais tranquila mais no começo assim foi horrivel, ela não olhava na minha cara e a minha mãe não olha na cara, tipo, nossa fiquei durante 2 semanas... do dia que começo sem se fala, sem olha na cara, nenhum, nenhum tipo de contato assim sabe (Carolina, 18, Ponta Grossa).

En los estudios sobre la situación de lesbianas y gays en relación con sus familias, el hogar familiar acostumbra a ser un lugar dónde se experimentan las consecuencias negativas de su orientación sexual (VALENTINE et altri, 2003), mientras que la casa propia se asocia a un lugar de bienestar y de identificación (ver ELWOOD, 2000; KENTLYN， 2008; GORMAN-MURRAY, 2007) y hasta un lugar que permite a parejas de lesbianas subvertir los roles tradicionales de género en relación al trabajo doméstico y de cuidados (BARRETT, 2015). A pesar de que en las entrevistas realizadas se han encontrado casos que demuestran que la casa familiar también puede ser un lugar de apoyo, demostrando que el hogar familiar no tiene porque ser siempre un lugar homofóbico (GORMAN-MURRAY, 2008), en la mayoría de los casos, sí que se concibe el hogar como un espacio de conflicto para jóvenes no heterosexuales.

Los ejemplos de Larissa, Adriana y Larissa muestran al hogar familiar como un espacio de malestar, y las restricciones que sufren en él se extienden al espacio público. La separación entre lo público y lo privado se vuelve ambigua, ya que la homofobia que se sufre en casa se extiende al espacio público impidiendo que puedan mostrarse como lesbianas en él y deban ocultar siempre la relación con su pareja.

La sexualidad aquí se enlaza con la edad y la clase de manera que intensifica esta situación: la dependencia económica (Larissa afirma que 'depende' de sus padres) y emocional (como muestra la cita de Carolina) las atan a sus casas y su sostenimiento 
depende de su relación con sus familias.

Este tipo de situaciones muestra como el uso y el significado que se da al espacio público está condicionado por la experiencia en el espacio privado. Uno no se entiende sin el otro. Muchas feministas han mostrado como el hogar puede ser un lugar de violencia y malestar, a pesar de las connotaciones positivas que en geografía se han asociado al hogar como un espacio de identificación (ver ROSE, 1993). Pero como crítica también a una visión (blanca) muy negativa con las implicaciones del hogar, desde el feminismo negro también se ha cuestionado el hecho de que solo se entienda la casa como un lugar de opresión para las mujeres ya que para muchas mujeres negras el hogar significó un espacio de resistencia y reafirmación (hooks, 1990). En estos casos, la visión heteronormativa y adultocéntrica de lo que significa el hogar como lugar puede invisibilizar situaciones como las que viven estas mujeres jóvenes lesbianas. Sin pretender extrapolar estos resultados, este estudio apunta hacia la necesidad de tener en cuenta determinados colectivos que quedan en los márgenes y que dan visiones contradictorias, o al menos diferentes, de lo que puede significar un lugar tan relevante para la vida cotidiana como el hogar.

Sea cual sea la relación concreta en la casa familiar, lo que se muestra es como la relación entre los dos espacios es fundamental para definir la experiencia que se tiene en ellos. Como afirma Pateman:

Lo privado o personal y lo público o político se entienden como cosas separadas y como irrelevantes la una para la otra; la experiencia cotidiana de las mujeres confirma esta separación $\mathrm{y}$, simultáneamente, la niega y afirma la conexión integral entre las dos esferas. La separación de lo privado de lo público es parte de nuestras vidas y es a la vez la mistificación ideológica de la realidad liberal - patriarcal (PATEMAN, 1983, p. 131)

Esta separación entre lo público y lo privado que comenta la autora es lo que ha llevado también a pensar la ciudad como espacio público, relegando las casas a lo privado. Pero, si contabilizáramos el espacio que ocupan en una ciudad por un lado las plazas y por el otro todos los comedores de las casas, ¿qué tipo de espacio sería mayoritario en las ciudades? Seguramente en una ciudad hay más metros de comedores, dormitorios y cocinas que metros de calles y plazas. Pero a menudo las geografías urbanas y las discusiones sobre el Derecho a la Ciudad pasan por alto que el interior de las casas, y las relaciones que ocurren en ellas, también son parte de las ciudades, las forman. Fenster (2005) afirma que las relaciones de género, en relación con otras formas de opresión, limitan el acceso a la ciudad de las mujeres y argumenta que el hecho de aislar la discusión sobre el derecho a la ciudad del derecho al hogar crea una esfera pública neutral esterilizada de las relaciones de poder. Y el caso de estas chicas en Manresa y Ponta Grossa pone en evidencia que no solo importan las relaciones en el espacio privado porque son parte de sus vidas cotidianas en sus ciudades, sino porque también repercuten en la forma en cómo viven los espacios públicos. Entonces, el espacio público se convierte en una extensión de su casa. No existe tal separación rígida en sus vidas porque las limitaciones que se dan dentro del hogar por la mezcla de su sexualidad, su edad, su género, su condición económica y su etnicidad persisten cuando salen a la calle. Con su caso, se ejemplifica la paradoja que nos muestra Pateman y se muestra a la vez la importancia de tener en cuenta esta (no)separación entre espacios al 
¿Quién tiene Derecho a la Ciudad? Jóvenes

lesbianas en Brasil y Cataluña desde las

geografías emocionales e interseccionales

conceptualizar el Derecho a la Ciudad. Nos recuerda que las ciudades también están hechas de hogares y que lo que ocurre en ellos no puede separarse de los análisis sobre el espacio urbano. Como lo global repercute en lo local, lo privado repercute en lo público, y viceversa.

\section{Diferencias Geográficas a través de la Interseccionalidad}

Como se ha visto, uno de los grandes de los resultados más relevantes de esta investigación son las grandes semejanzas en las limitaciones de acceso al espacio público por razón de sexualidad. Si bien podrían apreciarse diferentes niveles de intensidad, la muestra no es lo suficientemente completa como para determinarlo. A pesar de esto, se han encontrado algunas diferencias significativas de acceso que precisamente no tienen tanto que ver con su orientación sexual, como con sus otras posiciones en estructuras de poder y en la existencia o no de determinados lugares.

Una de las diferencias más relevantes es en relación al género y sus implicaciones para el acceso a los espacios. En las narrativas de las chicas de Manresa el trabajo doméstico y de cuidados como actividad era ausente de sus narrativas. Prácticamente ninguna tenía responsabilidades familiares o eran muy circunstanciales. En cambio, las chicas de Ponta Grossa mostraban una carga importante de este tipo de trabajo estrechamente relacionado con los roles de género impuestos. Esto se visibiliza en los Relief Maps como el de Denise (Figura 1) y el de Ana (Figura 3) en los que se puede ver como sienten malestar en su casa (casa de su familia) no solo por su sexualidad (punto azul claro) sino también por su género (punto naranja). Hay otros ejemplos que apuntan hacia la misma dirección.
Eu vejo pelo meu irmão meu irmão não faz nada em casa e eu já tenho que ajuda então, ele fala assim "mas você... eu falo mas meu irmão não faz nada em casa por que que eu tenho que faze? "não mas você é mulher" entendeu? Então eles sempre falam isso (Larissa, 18 , Ponta Grossa)

Casa onde moro... acho que eu tenho obrigação de fazer algumas tarefas... moro com dois homens $e$ ainda sou mais nova né. (Ana, 24, Ponta Grossa)

Eu já terminei de estuda né, to procurando um serviço agora, mais eu só fico em casa assim, só saio final de semana ou treina terça, quinta e sábado com o meu time né e cuido da minha irmã pra minha mãe, esse é o favor que eu faço pra minha mãe (Lorena, 23, Ponta Grossa)

Eu tenho irmãos mais quem faz as coisas de casa mesmo só eu então. (Natalia, 24, Ponta Grossa)

Este tipo de tareas, como muestran las citas, son realizadas por ellas por el hecho de ser mujeres, y para algunas de ellas implica quedarse mucho tiempo en el espacio privado cuidando de hermanos más pequeños. La división sexual del trabajo, sería una de las diferencias encontradas que limita de forma diferente el acceso a los espacios, en este caso encerrándolas en el espacio privado por tener que hacer trabajo de cuidados.

Siguiendo con las relaciones de género y sus implicaciones, se encontró también una importante diferencia en el uso del espacio público en relación al miedo. Si bien las chicas en Manresa afirmaban sentir miedo en 
diferentes espacios de la ciudad, especialmente de noche y si volvían solas a sus casas, en Ponta Grossa este tipo de narrativas no son tan presentes. Analizando sus usos del espacio, la ausencia de miedo en las chicas de Ponta Grossa, se atribuye a su relativo uso del espacio público. Si bien para las manresanas las calles y plazas son un lugar que usan intensivamente (para pasear, sentarse a charlar o a beber), las pontagrossenses lo usan más como lugar de paso. Sin ser el tema central de este artículo, sí podría apuntarse a algunas posibles explicaciones, como el mayor índice de criminalidad en Ponta Grossa, que prevendría a las chicas a salir, la ordenación territorial misma y sus facilidades de movilidad o cuestiones culturales en relación al uso y función del espacio público.

A parte de las cuestiones relacionadas con el género, también existe otro factor con importantes repercusiones espaciales: la religión. En la mayoría de los Relief Maps de las chicas de Ponta Grossa aparece la iglesia como un lugar, y a menudo como lugar de opresión por ser lesbianas. El análisis de la religión en relación a la sexualidad escapa también el objetivo de esta investigación, pero cabe apuntar que solo la presencia de estos lugares ya es indicativo, ya que ninguna chica de Manresa siquiera mencionó las iglesias, centros de culto o religión en sus narrativas. La religiosidad pues, elemento muy relevante en Brasil y prácticamente inexistente entre la juventud catalana ${ }^{5}$ sería otra de las diferencias que implica limitaciones diversas de acceso a determinados espacios.

Estas diferencias muestran no solo que la localización geográfica es importante para su experiencia cotidiana de los espacios, sino también que sus otras posiciones e identidades son relevantes en sus vivencias. Aquí se ha mostrado como, junto con la sexualidad, el género es un condicionante muy relevante. De hecho, en los Relief Maps que realizaron las entrevistadas, las líneas que marcaban malestares más acentuados (los puntos estaban situados en la parte superior del mapa) y más estructurales (que eran presentes en muchos de los lugares que frecuentan) eran precisamente la sexualidad y el género. Aquí también ha aparecido la religión como un factor importante ya que incluye elementos espaciales muy directos como la presencia o no de espacios de culto. Otras cuestiones como la clase social o la raza se identificaron como muy relevantes en narrativas concretas y para espacios determinados, pero la muestra no permite identificar diferencias o semejanzas entre Manresa y Ponta Grossa ${ }^{6}$.

\section{Conclusiones}

La investigación sobre un grupo social en los márgenes (mujeres, lesbianas, jóvenes, de ciudades medianas) permite ver cómo el Derecho a la Ciudad, para dar cuenta de las exclusiones existentes, debe conceptualizarse de forma interseccional y emocional. Las discriminaciones que limitan éste derecho son múltiples e interseccionales. Una mirada que solamente tenga en cuenta la presencia o ausencia de determinados colectivos en el espacio público no alcanzan para dar cuenta de las formas actuantes de la desigualdad: el heterosexismo no solamente implica impedir la presencia sino hacerla invisible. Se ha mostrado que la dimensión emocional es necesaria para identificar ciertos tipos de discriminaciones y que además se deben tener en cuenta las diversas posiciones en las estructuras de poder. Análisis centrados en solo un tipo de opresión, como la clase o el género, construyen falsos neutros que invisibilizan otras opresiones, esconden la complejidad de las dinámicas urbanas y no dan cuenta de las problemáticas, y las posibilidades, que conllevan las ciudades. Por 
último, se ha mostrado también como el análisis de las relaciones sociales en los espacios privados es imprescindible para comprender las limitaciones que se dan en los espacios públicos.

\section{Todas las traducciones de citas del inglés al} castellano son propias.

2 Todos los nombres han sido modificados para preservar el anonimato.

3 Para una descripción y análisis más detallada de esta herramienta, ver Rodó-de-Zárate, 2014.

$4 \quad$ Para un análisis sobre el 'passing' en relación a este mismo grupo estudiado ver Rodó-de-Zárate, en prensa.

5 En la última encuesta a la juventud en Cataluña, un $80,2 \%$ de la juventud (entre 15 y 30 años) afirmo no asistir nunca a actos religiosos. Datos extraídos de la "Enquesta a la Joventut de Catalunya 2012. Principals Resultats Estadístics". Generalitat de Catalunya. 2013.

\footnotetext{
6 Para un análisis interseccional más detallado sobre las experiencias interseccionales que incluye la raza y la clase de forma específica en de jóvenes lesbianas en Manresa ver Rodó-de-Zárate 2013.
}

\section{Referências}

BARCELOS SOLIVA, Thiago. A Rua e o Medo: Algumas Considerações sobre a Violência Sofrida por Jovens Homossexuais em Espaços Públicos. Revista Latinoamericana de Geografia e Gênero, v. 2, n. 1, p. 92-103, 2011

BARRETT, Carla. Lesbians at Home: Gender and Housework in Lesbian Coupled Households. En: BROWNE, Kath; FERREIRA, Eduarda. Lesbian Geographies. Gender, place and power. Burlington: Ashgate, 2015, p. 55 - 70.
BELL, David; VALENTINE, Gill (Eds). Mapping desire: geographies of sexualities. London: Routledge, 1995.

BELL, David; BINNIE, John; CREAM, Julia; VALENTINE, Gill. All hyped up and no place to go. Gender, Place and Culture, v. 1, p. $31-47,1994$.

BINNIE, John. Coming out of geography: towards a queer epistemology? Environment and Planning D: Society and Space, v. 15, p. $223-37,1994$.

BONDI, Liz; DAVIDSON, Joyce; SMITH, Mick. Introduction: Geography's Emotional Turn. In: DAVIDSON, Joyce; BONDI, Liz; SMITH, Mick. Emotional Geographies. Aldersohot: Ashgate. 2007, p. 1 - 16.

BROWNE, Kath. Lesbian geographies. Social \& Cultural Geography, v. 8, n. 1, p. $1-7,2007$.

BROWNE, Kath; FERREIRA, Eduarda. Lesbian Geographies. Gender, place and power. Burlington: Ashgate, 2015.

CATTAN, Nadine; LEROY, Stéphane. La ville négociée : les homosexuel(le)s dans l"espace public parisien. Cahiers de géographie du Québec, v. 54, n. 151, p. 9 24, 2010.

COLLINS, Patricia Hill. Black Feminist Thought: Knowledge, Power and the Politics of Empowerment. Boston: Unwin Hyman, 1990.

DAVIDSON, Joyce; BONDI, Liz; SMITH, Mick. Emotional Geographies, Aldersohot: Ashgate, 2007.

DAVIS, Kathy. Intersectionality as Buzzword: A Sociology of Science 
Perspective on What Makes a Feminist Theory Successful. Feminist Theory, v. 9, n. 1, p. $67-85,2009$.

DOWNING, Garry. Virtual youth: nonheterosexual young people"s use of the internet to negotiate their identities and sociosexual relations. Children"s Geographies, v. 11, n. 1, p. $44-58,2013$.

ELWOOD, Sarah. Lesbian Living spaces: Multiple Meanings of home. Journal of Lesbian Studies, v. 4, n. 1, p. 11 - 27, 2000. FENSTER, Tovi. The right to the gendered city: different formations of belonging in everyday life. Journal of Gender Studies, v. $14, .3$, p. $217-231,2005$.

FERNANDES CARVALHAES, Flavia (et altri). Territórios, Gerações \& Cultura: (Des)continuidades das Expressões de Gênero entre Lésbicas. Revista Latinoamericana de Geografia e Gênero, v. 2, n. 1, p. $92-103,2011$.

GORMAN-MURRAY, Andrew. Contesting domestic ideals: queering the Australian home. Australian Geographer, v. 38, p. 195 $-213,2007$.

GORMAN-MURRAY, Andrew. Reconciling self: gay men and lesbians using domestic materiality for identity management. Social \& Cultural Geography, v. 9, n. 3, p. 283 301, 2008.

HARDING, Sandra. The Feminist Standpoint Theory Reader. New York and London: Routledge. 2004.

HARVEY, David. The Right to the City. New Left Review, v. 53, p. 23 - 40, 2008.

hooks, bell. Yearning: Race, Gender, and Cultural Politics. Boston: South End Press,
1990.

HYAMS, Melissa. Adolescent Latina Bodyspaces: Making Homegirls, Homebodies and Homeplaces. Antipode, v. 35, p. 535 - 558, 2003.

KENTLYN, Sue. The Radically Subversive Space of the Queer Home: 'Safety House'and 'Neighbourhood Watch, Australian Geographer, v. 39, n. 3, p. 327 - 337, 2008.

KUHAR, Roman. The Heteronormative Panopticon and the Transparent closet of the Public Space in Slovenia. In: KULPA, Robert; MIZIELIŃSKA, Joanna. Decentring western sexualities. Farham, Surrey: Ashgate, 2011, p. 149 - 166.

LANE, Nikki. All the Lesbians are White, All the Villages are Gay, but Some of Us are Brave: Intersectionality, Belonging, and Black Queer Women's Scene Space in Washington, D.C. In: BROWNE, Kath; FERREIRA, Eduarda. Lesbian Geographies. Gender, place and power. Burlington: Ashgate, 2015, p. $219-242$.

LEFEBVRE, Henrie. Le droit à la ville. Paris: Anthropos, 1968.

MCCALL, Leslie. The Complexity of Intersectionality. Signs: Journal of Women in Culture and Society, v. 30, p. $1-30$, 2005.

MULLER MYRDAHL, Tiffany Muller. Ordinary (small) cities and LGBQ lives." ACME: An international journal for Criitical Geographies, v. 12, n. 2, p. $279-$ 304, 2013.

PATEMAN, Carole. Feminist Critiques of the Public/Private Dichotomy. In: BENN, Stanlei; GAUS, Gerald (eds.) Public and Private in Social Life. New York, NY: St. 
Martin’s Press, 1983, p. 153 - 160.

PEAKE, Linda. Race' and sexuality: challenging the patriarchal structuring of urban social space. Environment and Planning D: Society and Space, v. 11, p. $415-432,1993$.

PLATERO, Lucas (Ed). Intersecciones: cuerpos y sexualidades en la encrucijada. Barcelona: Edicions Bellaterra, 2012.

PODMORE, Julie. Lesbians in the Crowd: gender, sexuality and visibility along Montréal's Boul. St- Laurent, Gender, Place \& Culture, v. 8, n. 4, p. 333 - 355, 2001.

PODMORE, Julie. Gone 'underground'? Lesbian visibility and the consolidation of queer space in Montréal. Social \& Cultural Geography, v. 7, n. 4, p. 595-625, 2006.

PODMORE, Julie. Contested Dyke Rights to the City: Montréal's 2012 Dyke Marches in Time and Space. En: BROWNE, $\mathrm{K}$ and FERREIRA, E. Lesbian Geographies. Gender, place and power. Burlington: Ashgate, 2015, p. $71-90$.

PRITCHARD, Annette; MORGAN, Nigel; SEDGLEY, Diane. In search of lesbian space? The experience of Manchester's gay village. Leisure Studies, v. 21, n. 2, p. 105123, 2002.

PURCELL, Mark. Excavating Lefevbre: The right to the city and its urban politics of the inhabitant. GeoJournal, v. 58, p. $99-108$, 2002.

RODÓ-DE-ZÁRATE, Maria. Young lesbians negotiating public space in Manresa: an intersectional approach through places. Children's Geographies, v. 13, n. 4, p. 413 434, 2013.
RODÓ-DE-ZÁRATE, Maria. Developing geographies of intersectionality with Relief Maps: reflections from youth research in Manresa, Catalonia. Gender, Place \& Culture, v. 21, n. 8, p. 925 - 944, 2014.

RODÓ-DE-ZÁRATE, Maria. Metodologías feministas visuales para el estudio de la interseccionalidad. Actas del XXIII Congreso de la Asociación de Geógrafos Españoles 459-68, 2014 b.

RODÓ-DE-ZÁRATE, Maria. Affective inequality and heteronormative discomforts. Tijdschrift voor Economische en Sociale Geografie [Journal for Economic and Social Geography] (en prensa).

ROSE, Gillian. Feminism \& geography. Cambridge: Polity Press, 1993.

SANTOS SOLLA, Xosé. Espacios disidentes en los procesos de ordenación territorial" Documents d'Anàlisi Geogràfica, v. 40, p. $69-104,2002$.

SILVA, Joseli Maria (Ed.). Geografias subversivas: discursos sobre espaço, gênero e sexualidades. Ponta Grossa: Todapalavra, 2009.

SILVA, Joseli Maria; ORNAT, Mario Jose. Intersectionality and transnational mobility between Brazil and Spain in travesti prostitution networks. Gender, Place \& Culture: A Journal of Feminist Geography, v. 22, n. 8, p. $1073-1088,2015$.

TAYLOR, Yvete; HINES, Sally; CASEY, Mark. Theorizing Intersectionality and Sexualities. Houndmills, Basingstoke, Hampshire: Palgrave Macmillan, 2011.

VALENTINE, Gill. (Hetero)sexing space: lesbian perceptions and experiences of 
¿Quién tiene Derecho a la Ciudad? Jóvenes

lesbianas en Brasil y Cataluña desde las

geografías emocionales e interseccionales

everyday spaces. Environment and Planning D: Society and Space, v. 11, n. 4, p. 395 - 413, 1993.

VALENTINE Gill. Children should be seen and not heard: The production and transgression of adults' public space. Urban Geography, v. 17, p. 205 - 220, 1996.

VALENTINE, Gill; SKELTON, Tracey. Finding oneself, Losing oneself: The Lesbian and Gay 'Scene' as a Paradoxical Space. International Journal of Urban and Regional Research, v. 27 , n. 4, p. $849-66$, 2003. 